This item was submitted to Loughborough's Research Repository by the author.

Items in Figshare are protected by copyright, with all rights reserved, unless otherwise indicated.

\title{
Design methodology and performance of an indraft wind tunnel
}

PLEASE CITE THE PUBLISHED VERSION

http://www.raes.org.uk/aero_journal.asp

\section{PUBLISHER}

(c) Royal Aeronautical Society

\section{VERSION}

VoR (Version of Record)

\section{LICENCE}

CC BY-NC-ND 4.0

\section{REPOSITORY RECORD}

Johl, G., Martin A. Passmore, and Peter M. Render. 2010. "Design Methodology and Performance of an Indraft Wind Tunnel”. figshare. https://hdl.handle.net/2134/6674. 
This item was submitted to Loughborough's Institutional Repository (https://dspace.lboro.ac.uk/) by the author and is made available under the following Creative Commons Licence conditions.

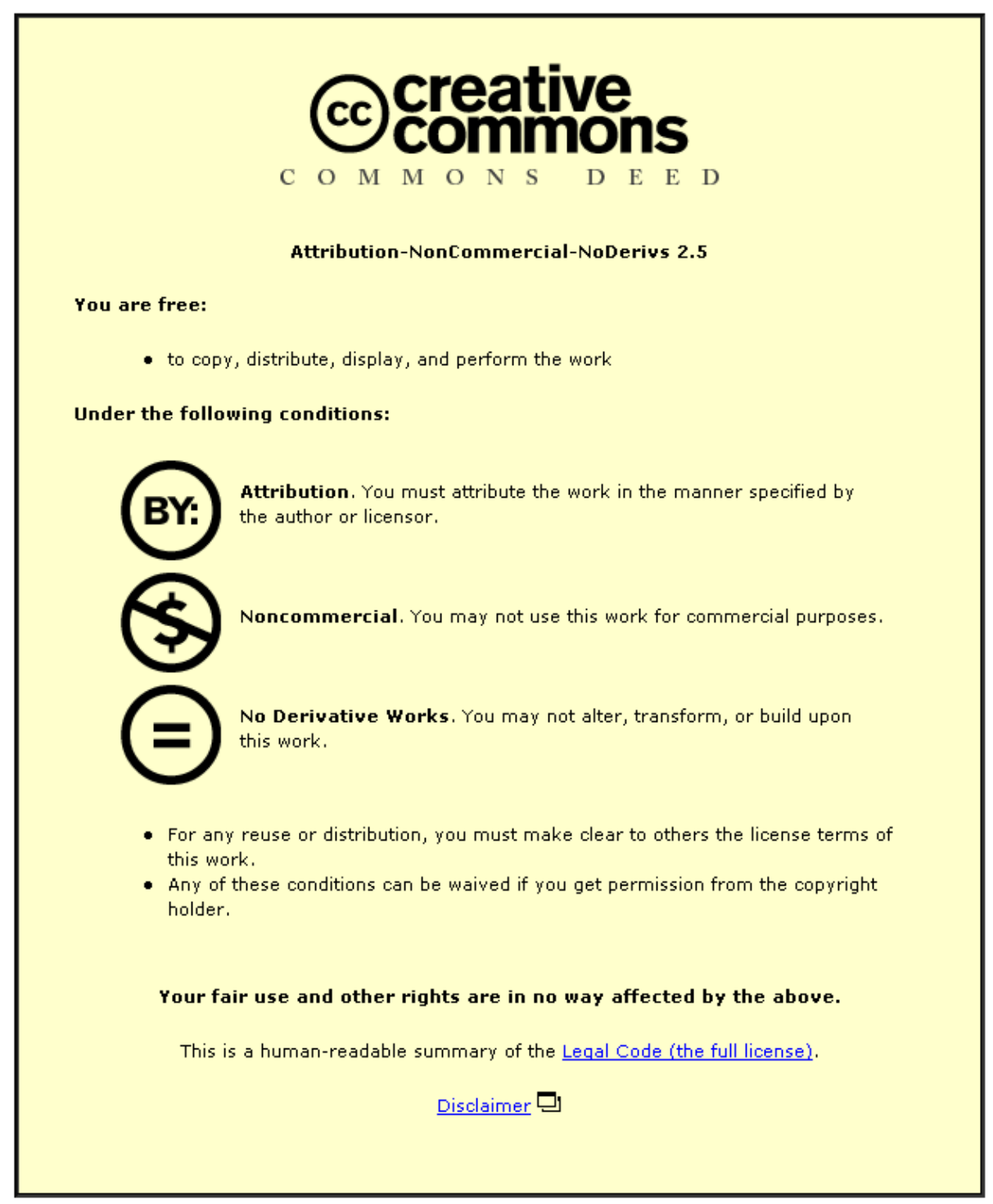

For the full text of this licence, please go to: http://creativecommons.org/licenses/by-nc-nd/2.5/ 


\title{
Design methodology and performance of an indraft wind tunnel
}

\author{
G. Johl, M. Passmore and P. Render \\ Department of Aeronautical and Automotive Engineering \\ Loughborough University \\ Loughborough, UK
}

\begin{abstract}
The design methodology and performance of Loughborough University's new $1.9 \mathrm{~m} \times 1.3 \mathrm{~m}$, indraft wind tunnel is discussed in the following paper. To overcome severe spatial and financial constraints, a novel configuration was employed, with the inlet and exit placed adjacent to each other and opened to atmosphere. Using a fine filter mesh, honeycomb, two turbulence reduction screens and a contraction ratio of $7 \cdot 3$, flow uniformity in the working area of the jet at $40 \mathrm{~ms}^{-1}$ is shown to be within $0 \cdot 3 \%$ deviation from the mean velocity, with turbulence intensity in the region of $0 \cdot 15 \%$. Working section boundary layer characteristics are shown to be consistent with that of a turbulent boundary layer growing along a flat plate, which originates at the point of inflection of the contraction. A maximum velocity of $46 \mathrm{~ms}^{-1}$ was achieved from a $140 \mathrm{~kW}$ motor, compared to a prediction of $44 \mathrm{~ms}^{-1}$, giving an energy ratio of $1 \cdot 42$. Comparison between theoretical and measured performance metrics indicate differences between the way modules perform when part of a wind tunnel system compared to data gathered from test rigs.
\end{abstract}

\section{NOMENCLATURE}

$\begin{array}{ll}A & \text { area } \mathrm{m}^{2} \\ A_{r} & \text { area ratio } \\ C & \text { chord mm } \\ C p_{r} & \text { static pressure recovery coefficient } \\ C R & \text { contraction ratio } \\ D & \text { diameter or hydraulic diameter mm } \\ E R & \text { energy ratio } \\ f_{u} & \text { axial turbulence reduction factor }\end{array}$

stagnation or total pressure $\mathrm{Nm}^{-2}$

local height or width of contraction measured from the centreline mm

local total pressure loss coefficient

component of screen loss coefficient due to open area

length $\mathrm{mm}$

screen mesh width mm

number of screens and power for contraction contour static pressure $\mathrm{Nm}^{-2}$

power $\mathrm{W}$

dynamic pressure $\mathrm{Nm}^{-2}$

radius or hydraulic radius $\mathrm{mm}$

Reynolds number

reserve factor

vane spacing $\mathrm{mm}$

turbulence intensity \%

instantaneous velocity ( $x$-component) $\mathrm{ms}^{-1}$

time averaged axial velocity $\mathrm{ms}^{-1}$

axial length co-ordinate mm

location of contraction matchpoint or point of inflection local width measured from the centreline of the working section $\mathrm{mm}$

local height measured from the floor of the working section mm

air density $\mathrm{kgm}^{-3}$

diffuser included angle and momentum thickness degrees momentum thickness mm

efficiency

screen fractional open area 
$\alpha \quad$ kinetic energy coefficient

$\psi \quad$ screen aperture mm

$\delta \quad$ boundary-layer thickness $\mathrm{mm}$

$\delta^{*} \quad$ displacement thickness mm

\section{Subscripts}

1 upstream of component

2 downstream of component

C contraction

HC honeycomb

L local

W wire

WS working section

\subsection{INTRODUCTION}

A significant number of new wind tunnels are designed for commercial organisations that understandably do not wish for the details behind their designs to be made public. Furthermore, little data exists on the performance of indraft wind tunnels since closed circuit designs are generally favoured, due to concerns over working section flow quality. Wherever possible, therefore, information on the design and performance of new wind tunnels should be published, in order to help future designers build on past successes and avoid costly mistakes. Although the wind tunnel presented in this paper is unremarkable in terms of its operating performance, it is unusual insofar as it is an indraft tunnel of novel configuration that has been designed from the outset to satisfy the often-conflicting requirements of both aeronautical and automotive testing, within severe spatial and financial constraints. Additionally, since most published data tends to focus on overall circuit performance, measured performance metrics for individual modules are also presented to promote understanding of how modules interrelate as this may differ to how they perform when tested in isolation in test rigs.

\subsection{Design objectives and constraints}

The wind tunnel needed to be able to accommodate automotive shapes of approximately $1 / 4$ scale, giving a length based Reynolds number of the order of two million at approximately $5 \%$ blockage based on frontal area. One quarter scale was deemed adequate for investigations of the underlying flow mechanisms and for basic research on simplified bluff and quasi-streamlined bodies. It was not intended to use the facility to conduct 'real' vehicle shape development where the detail optimisation (of edge radii, gurney flaps etc.) tends to be sensitive to Reynolds number. Such development is generally performed in excess of $40 \%$ scale in the racecar sector and at full scale by the large motor manufacturers.

The tunnel also needed to accommodate aeronautical half-models up to a semi-span of $1 \mathrm{~m}$ and chord of $0.3 \mathrm{~m}$. In order to achieve suitable test Reynolds numbers (Re), a maximum working section velocity greater than $40 \mathrm{~ms}^{-1}$ was required. The velocity variation in the working area of the jet was required to be less than $0 \cdot 3 \%$ deviation from the mean velocity(1), and it was considered desirable for working section turbulence intensity to be in the region of $0 \cdot 1 \%$ at $40 \mathrm{~ms}^{-1}$. The available space envelope measured $18 \mathrm{~m}$ (length) $\times 10 \mathrm{~m}$ (width) $\times 7.5 \mathrm{~m}$ (height), and maximum fan power consumption had to be less than $140 \mathrm{~kW}$. The budget for the tunnel structure, fan and balance was $£ 350,000$ (sterling).

\subsection{Preliminary closed circuit design}

Initially, a conventional closed circuit design was investigated, since design guidelines for such tunnels were well established(1-4). However, the requirement to test a $25 \%$ scale automotive model, effectively set the working section area at approximately $2 \cdot 5 \mathrm{~m}^{2}$, and preliminary investigations revealed that the resulting closed circuit tunnel was very cramped and ill proportioned, and therefore had a greater risk of experiencing secondary flows. The contraction ratio $(C R)$ was limited to $4 \cdot 5$, and although the desired working section turbulence intensity could be achieved with sufficient screens the risk of significant nonuniformity was considerable. Increasing the contraction ratio by using a wide-angle diffuser severely reduced the space available for the remaining tunnel modules, and the additional screens needed to prevent this diffuser from separating(3), increased costs. There were also insufficient funds for a heat exchanger, needed to remove the energy put into the airstream by the fan. Excluding the heat exchanger would result in a rise in tunnel air temperature during a test and make taking measurements, such as Hot Wire Anemometry, very difficult. For these reasons (and since the authors were prepared not to run the tunnel in heavy rain), the feasibility of employing an indraft configuration was investigated. The authors appreciated that a drawback of the indraft design was that the working section would be below atmospheric pressure, hence making it difficult (yet by no means impossible) to install boundary-layer bleed devices or a moving ground.

\subsection{Indraft design}

Removing corners three and four from the closed circuit design (corner 1 being the first corner downstream of the working section) created a previously unseen 'horseshoe' configuration, as shown in Figs 1 and 2 . This made for a better-proportioned wind tunnel, and effectively created a long diffuser downstream of the working section. The intake and exit of the tunnel were made to penetrate the walls of the wind tunnel building, exposing both to atmosphere. Consequently, the impact on working section turbulence intensity and flow uniformity, from gusts blowing into the intake was unknown. Additionally, a boiler house located $5 \mathrm{~m}$ opposite the intake and exit was likely to create a recirculation zone for the jet exiting the final diffuser, and cause interference effects with the intake flow. Since the extent of these problems could not be quantified prior to running the tunnel, allowance was made in the pressure loss calculations for the future inclusion of inlet louvres and exit guide vanes to deflect the flow away from the inlet. Details of the internal circuit geometry are provided in Table 1.

\subsection{General description}

See Figs 1 and 2.

\subsection{Construction}

The wind tunnel was constructed from timber modules located on steel supports. Timber was chosen rather than steel as it was considered less likely to 'drum' and would be easier to work with when in-

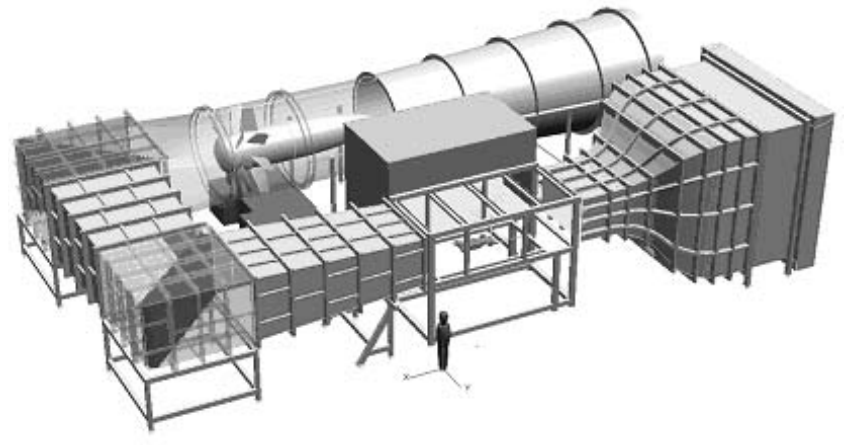

Figure 1. 3D Solid model. 


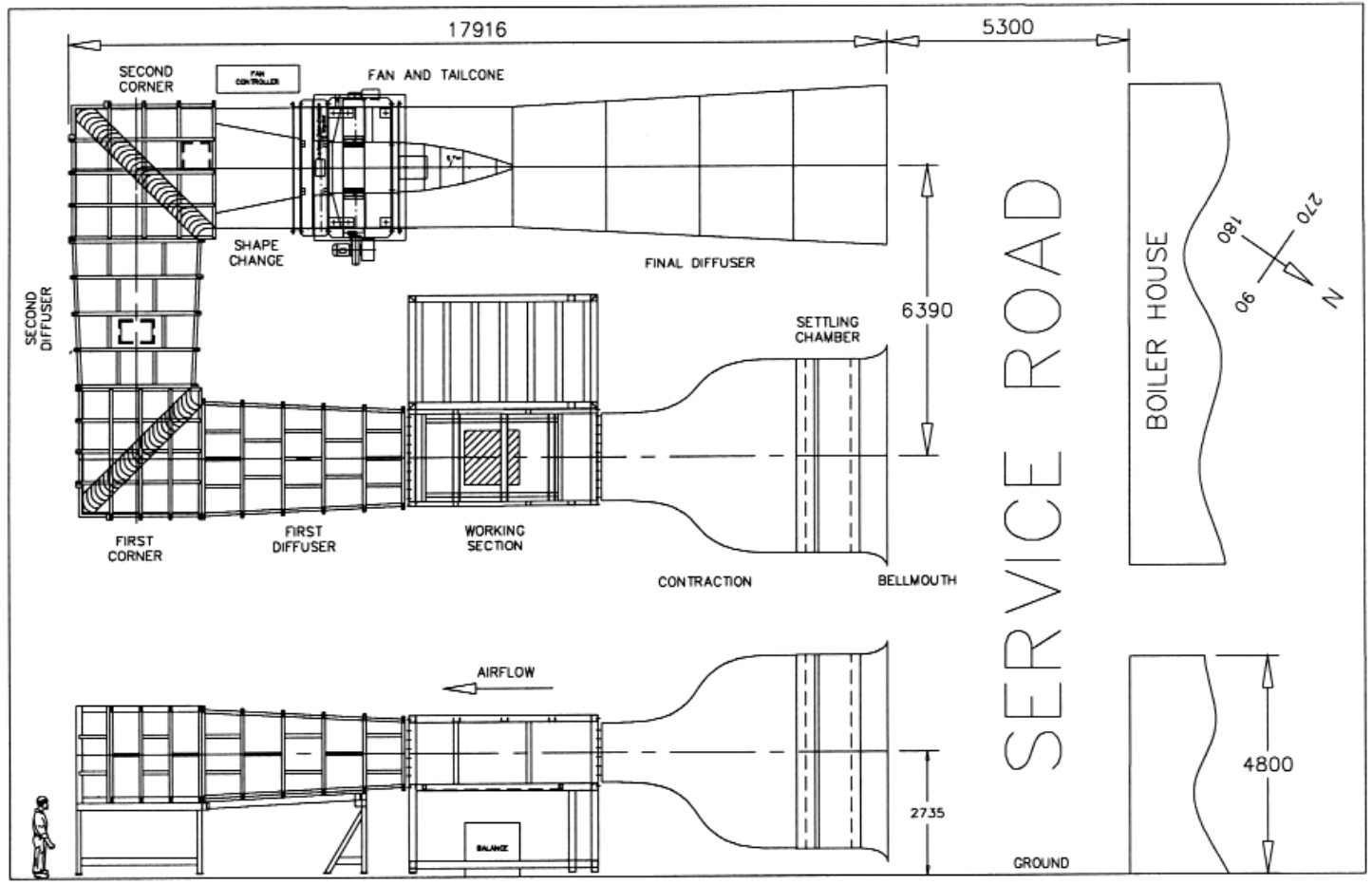

Figure 2. General arrangement (dimensions in $\mathrm{mm}$ ).

serting instrumentation. The final diffuser was manufactured from $5 \mathrm{~mm}$ thick steel, since a timber fabrication would have required a great deal of surrounding support structure. The shape-change was made from fibreglass on account of its complex geometry. The timber diffusers and corner modules were of box construction, typically manufactured from $25 \mathrm{~mm}$ plywood, with $140 \mathrm{~mm} \times 70 \mathrm{~mm}$ end flanges and similarly dimensioned battening spaced every $500 \mathrm{~mm}$.
The internal surfaces were sanded, cleaned and then varnished. The support tables and working section carcass were typically constructed from $150 \mathrm{~mm} \times 100 \mathrm{~mm} \times 5 \mathrm{~mm}$, hollow steel box section for rigidity, with adjustable load feet to allow vertical adjustment. After final positioning the support tables were bolted to the laboratory floor.

Table 1

Internal dimensions at module inlet

\begin{tabular}{|c|c|c|c|c|c|c|c|c|}
\hline Module & $\begin{array}{l}\text { Width } \\
(\mathrm{mm})\end{array}$ & $\begin{array}{l}\text { Height } \\
(\mathrm{mm})\end{array}$ & $\begin{array}{l}\text { Length } \\
(\mathrm{mm})\end{array}$ & $\begin{array}{l}\text { Corner } \\
\text { Fillet (mm) }\end{array}$ & $\begin{array}{l}D \\
(m m)\end{array}$ & $A\left(m^{2}\right)$ & $\begin{array}{l}\text { Area } \\
\text { Ratio(Ar) }\end{array}$ & $\begin{array}{l}\mathrm{R} \\
(\mathrm{mm})\end{array}$ \\
\hline Bellmouth & 4820 & 4820 & 600 & - & $F$ & $23 \cdot 232$ & $1 \cdot 274$ & 2719 \\
\hline Settling chamber & 4270 & 4270 & 1390 & - & - & $18 \cdot 233$ & - & 2409 \\
\hline Contraction & 4270 & 4270 & 4980 & $F$ & - & $18 \cdot 233$ & $7 \cdot 324$ & 2409 \\
\hline Working section & 1920 & 1320 & 3600 & 150 & $F$ & $2 \cdot 489$ & - & 890 \\
\hline First diffuser & 1940 & 1320 & 4450 & 150 & - & $2 \cdot 516$ & 1.584 & 895 \\
\hline First corner & 2340 & 1780 & - & 300 & - & 3.985 & - & 1126 \\
\hline Second diffuser & 2340 & 1780 & 3250 & 300 & - & 3.985 & 1.384 & 1126 \\
\hline Second corner & 2630 & 2180 & - & 330 & - & $5 \cdot 516$ & - & 1325 \\
\hline Shape change & 2630 & 2180 & 1835 & 330 & - & $5 \cdot 516$ & - & 1325 \\
\hline Fan & - & - & 4640 & - & 2650 & $5 \cdot 515$ & - & 1325 \\
\hline Final diffuser & - & - & 8195 & F & 2658 & 5.549 & 1.744 & 1329 \\
\hline Exit mesh & - & - & - & - & 3510 & 9.676 & - & 1755 \\
\hline
\end{tabular}




\subsection{DESIGN METHODOLOGY}

\subsection{Bellmouth intake}

A bellmouth is used to ingest ambient air and guide it into the settling chamber without separation. An elliptic bellmouth with an axis ratio of $2 \cdot 18$ was chosen, since empirical data had shown this to result in full flow attachment at impingement incidences of up to 25 degrees $(5)$.

\subsection{Settling chamber}

The function of the settling chamber is to produce a parallel, spatially uniform, steady stream of air. In general, honeycomb is used to straighten the flow and suppress lateral turbulence, while screens are used to reduce spatial variations in the axial velocity and reduce axial turbulence. While screens do act to turn the flow normal to the plane of the screen, they are not as effective a flow straighter as honeycomb, which is why the two are usually used in combination ${ }^{(2)}$. The screen axial turbulence reduction factor $f_{u}$, is defined as being the ratio between the downstream and upstream root-mean-square (RMS) of the instantaneous velocity, $u^{(2,6-7)}$ :

$$
\begin{aligned}
& f_{u}=\sqrt{\overline{u_{2}^{2}}} / \sqrt{\overline{u_{1}^{2}}} \\
& f_{u}=\left(\frac{1}{\left(1+K_{L}\right)}\right)^{n}
\end{aligned}
$$

It may therefore be seen that multiple screens $(n>1)$ are more effective than a single screen of the same overall pressure drop coefficient, $K_{L}$, which is defined as(7):

$$
\begin{gathered}
K_{L}=K_{o}+\frac{55 \cdot 2}{\mathrm{Re}_{\mathrm{W}}} \\
K_{o}=\left(\frac{1-0 \cdot 95 \beta}{0 \cdot 95 \beta}\right)^{2} \\
\beta=\left(\frac{\psi^{2}}{\left(\psi+D_{W}\right)^{2}}\right)
\end{gathered}
$$

Where $K_{o}$ is the pressure loss coefficient due to screen open area, $\mathrm{Re}_{\mathrm{W}}$ is Reynolds number based on wire diameter, $\beta$ is fractional screen open area, $\psi$ is aperture, and $D_{W}$ is wire diameter. A singlepiece filter mesh of $D_{W}=0.31 \mathrm{~mm}$, and $\psi=0.52 \mathrm{~mm}$, was located 200mm downstream of the bellmouth to keep out small particles. A $5 \mathrm{~m}$ wide service road in front of the intake provides access for cleaning this mesh. Since wake coalescence and consequent flow unsteadiness tends to occur for screens with open areas, $\beta<57 \%(8)$, and the open area of the filter mesh was only $39 \%$, a mixing length of 700mm was allowed between the filter mesh and the honeycomb. This gap was also intended to house louvres should they prove necessary during commissioning.

Hexagonal aluminium honeycomb with a cell hydraulic diameter, $D_{H C}=9 \cdot 525 \mathrm{~mm}$, was located at the end of the mixing length. A cell length to diameter ratio $\left(L_{H C} / D_{H C}\right)$ between six to 12 (12 being for fully developed flow) is recommended(6,7) although there is no real data to support where it should specifically be within this range. Since the honeycomb effectively acts as a low pass filter, with $D_{\mathrm{HC}-}$ constraining the lateral dimension of the largest eddy within the honeycomb, it may be argued that $D_{H C}$ should be less than the integral length scale of the turbulent eddies present upstream of the honeycomb. However, since the integral length scales were unknown $D_{H C}$ $=9.525 \mathrm{~mm}$ was chosen as this had been used to good effect in previous wind tunnels. Producing fully developed exit flow was not desired, since such long honeycomb would actually generate axial turbulence which would then have to be removed by the screens. Commercially available support steelwork resulted in an $L_{H C} / D_{H C}$ of 8.7 being used.

The wind tunnel was fitted with two, single-piece turbulence reduction screens. A coarse screen $\left(D_{W}=0.6 \mathrm{~mm} ; \psi=1.9 \mathrm{~mm} ; \beta=\right.$ $59 \%)$ was placed immediately downstream of the honeycomb, to suppress honeycomb-generated turbulence $(6)$. A fine screen $\left(D_{W}=\right.$ $0.4 \mathrm{~mm} ; \psi=1.29 \mathrm{~mm} ; \beta=57 \%$ ) was located 80 mesh widths, $M$ (where $M=\psi+D_{W}$ ) downstream of the coarse screen, since this was in excess of the minimum of $25 \mathrm{M}$ recommended for the small scale turbulence generated by a screen operating in the supercritical regime to decay below incoming levels ${ }^{(8)}$. A conservative settling length of $118 M(200 \mathrm{~mm})$ was allowed downstream of the second screen prior to the contraction.

For installation, the opposing sides of each screen were sandwiched between $2.5 \mathrm{~mm}$ thick stainless steel hook strips and then TIG welded along the edge. The hook strips were hung on parallel flange channel tensioning bars and bolts used to push the bars out to tension the screens.

In order to reduce the dynamic loads and total pressure losses on screens and honeycombs, the cross sectional area of the settling chamber is usually made as large as possible (while considering the spatial requirements of other modules). This was achieved by employing a contraction between the settling chamber and the working section.

\subsection{Contraction}

In addition to improving circuit efficiency by reducing losses in the settling chamber, a contraction is used to improve flow uniformity and reduce the turbulence intensity in the working section. A review of literature concerning contraction design ${ }^{(9)}$, shows that most of the work is concerned with two dimensional or axisymmetric contractions, whereas most practical contractions are three-dimensional and of rectangular cross section. The reviewer states that: “...with little regard for design criteria and real conditions in wind tunnel contractions, these solutions are tools of flow analysis rather than methods of contraction design...” The designer of a three-dimensional wind tunnel contraction of rectangular cross section, has to address the following issues:

- Selection of the contraction ratio $(C R)$.

- Definition of corner fillet growth.

- Determination of the contraction length $\left(L_{C}\right)$.

- Decision on aspect ratio change.

- Definition of the contraction contour.

The selection of the contraction ratio is largely driven by the required working section turbulence intensity, $\mathrm{Tu}$, defined as:

$$
T u=\sqrt{\overline{u^{2}}} / U \%
$$

Where, $u$, is the instantaneous axial velocity and $U$, is the time averaged axial velocity. Since the $R M S$ velocity $\sqrt{\overline{u^{2}}}$ does not change appreciably during contraction ${ }^{(10)}$, the turbulence intensity is thereby reduced by the value of $C R$. The $C R$ of $7 \cdot 3$ used in this design, was the largest possible when coupled to a working section area of $2 \cdot 489 \mathrm{~m}^{2}$, whilst also allowing adequate space for the other tunnel modules. 
Although the bulk flow velocity increases along the length of the contraction, the wall velocity in a finite length contraction does not increase monotonically. Since the flow is subsonic the wall curvature at inlet to the contraction has an upstream effect and causes the streamlines in the parallel duct downstream of the last screen to curve,(11). For this reason, the end of the $200 \mathrm{~mm}$ settling length $(118 M)$ downstream of the last turbulence screen was placed $516 \mathrm{~mm}$ $\left(0 \cdot 107 D_{C}\right)$, upstream of the start of curvature of the contraction, where $D_{C}$ is the hydraulic diameter at inlet to the contraction. This streamline curvature produces a non-uniform velocity profile within this $516 \mathrm{~mm}$ parallel section, and the wall velocity reaches a local minimum (velocity undershoot) near the inlet of, but within, the contraction. A similar argument describing the behaviour of the boundary layer indicates the existence of a local wall-velocity maximum (velocity overshoot) near the exit of a finite length contraction(11), and it is this that accounts for the concave velocity profile of wind tunnel working sections.

Since separation in a rectangular contraction is most likely to occur in the corner region due to secondary flows caused by merging of the wall boundary layers ${ }^{(9)}$, 45-degree corner fillets were used to reduce the impact of these secondary flows. The fillets were designed to grow from nothing at inlet to the contraction, to their final value of $150 \mathrm{~mm} \times 150 \mathrm{~mm}$ at exit, according to the following cubic relation(12).

[Local Fillet Area $]=\left(3\left(x / L_{C}\right)^{2}-2\left(x / L_{C}\right)^{3}\right)$, Fillet Area at Exit $]$

A numerical investigation(9) into the effect of the relative length $L_{C} / D_{C}$, of a three-dimensional contraction of rectangular cross section, on exit flow uniformity, velocity overshoot and corner velocity distribution, showed that an $L_{C} / D_{C} \approx 1$, was a good design compromise, which was free from separation for a $C R$ of 9 . An $L_{C} D_{C}=1.03$ was used in this design and $L_{C}$ is shown in Fig. 3. This investigation also showed that there was no reason to follow the tradition of maintaining aspect ratio similarity along the length of the contraction in order to prevent the flow from distorting. Results also suggested that a square cross section was a good choice for the settling chamber no matter what the geometry of the working section. A contraction inlet measuring 4,270mm $\times 4,270 \mathrm{~mm}$ was therefore adopted, since restrictions on the available tunnel width meant that having a square settling chamber allowed a diffuser of larger area ratio to be used downstream of the fan. The following equations were used to generate contraction contours of matched curves ${ }^{(9)}$ :

$$
\begin{aligned}
& \left(J-J_{2}\right)\left(J_{1}-J_{2}\right)=1-\left[\left(x / L_{C}\right)^{n} / X^{n-1}\right] \quad 0 \leq x / L_{C} \leq X \quad \ldots \text { (8) } \\
& \left(J-J_{1}\right)\left(J_{2}-J_{1}\right)=1-\left[\left[1-\left(x / L_{C}\right)\right]^{n} /(1-X)^{n-1}\right] \quad X \leq x / L_{C} \leq 1
\end{aligned}
$$

Where, $J$, is the local height or width of contraction measured from the centreline; $X$, is the matchpoint between the two equations; and $n$, is the power used to produce the required radius of curvature. The use of large radii of curvature at inlet and exit of the contraction in order to alleviate adverse pressure gradients has been advocated(13), and graphical illustrations of the growth of the boundary layer along such a contour are also available ${ }^{(14)}$. Streamline curvature upstream of the contraction inlet causes a severe thickening of the boundary layer, which reaches its maximum thickness at some point just within the inlet. Local curvature effects then cause the near wall flow to be accelerated rapidly up to the point of inflection (or matchpoint, $X$ shown in Fig. 3), at which point the boundary layer attains its minimum thickness, and in some cases relaminarises.

Since the risk of boundary layer separation is greatest at the inlet, and exit uniformity is critical to models under test, these may be set as the two most important criteria by which to evaluate potential contours. Although the philosophy of having a short, steep, central section with large radii of curvature at the ends holds true, results from a numerical investigation show that combining a slightly lower power contour (smaller radius of curvature) upstream of the matchpoint with a higher power contour downstream, reduces the velocity

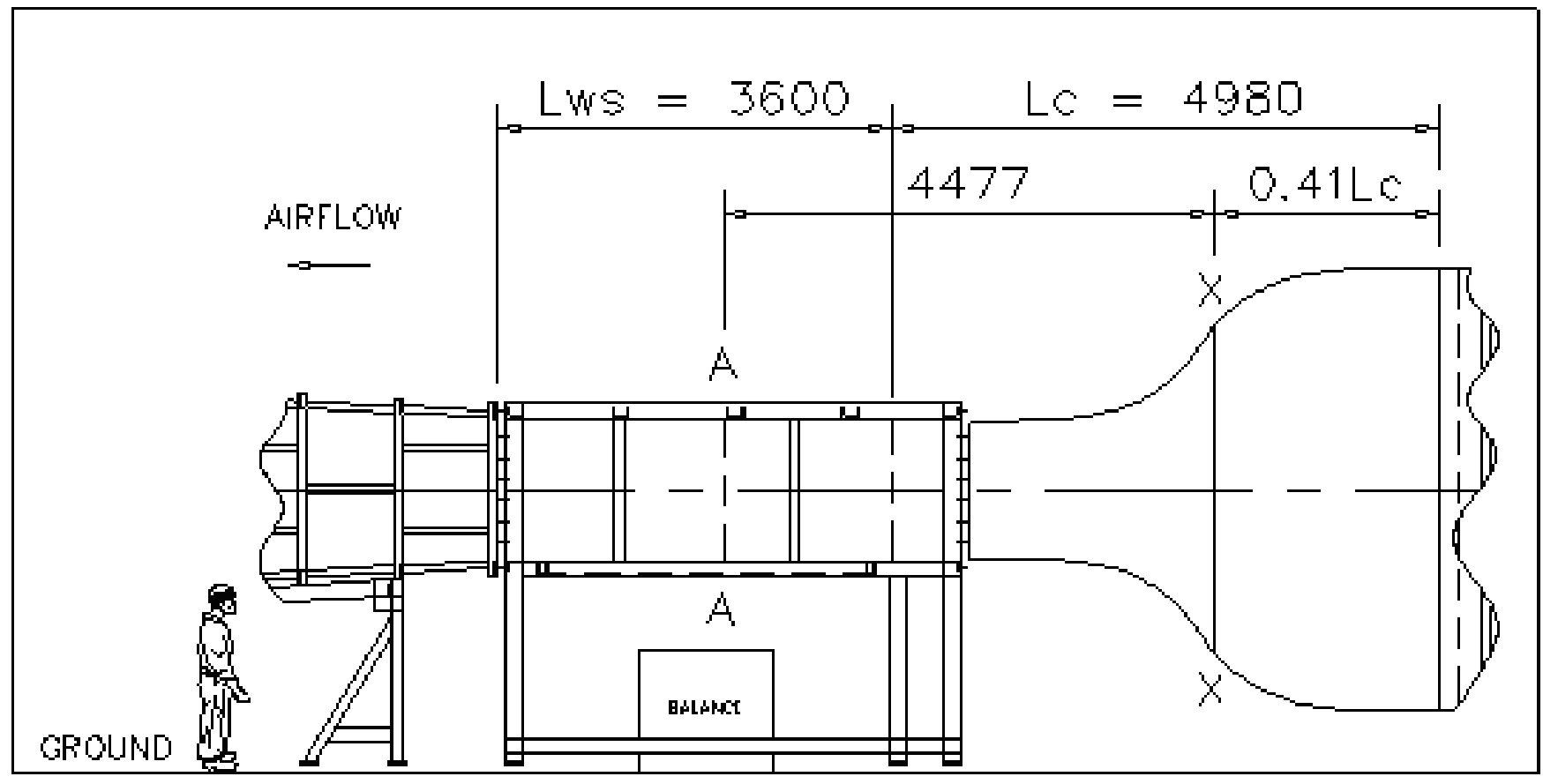

Figure 3. Side elevation of contraction and working section (dimensions in $\mathrm{mm}$ ). 
undershoot at inlet whilst improving exit uniformity by producing a parallel duct at the end of the contraction ${ }^{(9)}$. An upstream power, $n$, of four and a downstream power of six were therefore used to generate contours for this design, resulting in a parallel duct equal to one working section hydraulic radius. Matching the two different contours resulted in a discontinuity at the match point, which was eliminated by calculating the local gradients of both contours at either side of the matchpoint and making cuts at the two points where the gradients matched. A cubic spline was then fitted between these two points to produce a smooth transition. There is no reported advantage in having different matchpoint locations for the vertical and horizontal contours $(9,13)$, and so a matchpoint of $X=0 \cdot 41 L_{C}$ was selected since this had been shown to produce good exit flow uniformity(9). Despite the transition from square to rectangular geometry, the same values of $n$, were used for the roof/floor and sidewall contours.

The contraction was delivered in four sections, which were then bolted together on site. Each section comprised 18mm thick plywood formers overlaid with two layers of $3 \mathrm{~mm}$ thick plywood to form the internal skin. This method cost approximately $1 / 3$ that of a more traditional fibreglass construction, whilst making it much easier to achieve a smooth profile.

\subsection{Working section}

The inlet cross-section measures $1,920 \mathrm{~mm}$ (width) $\times 1,320 \mathrm{~mm}$ (height), and the exit measures $1,940 \mathrm{~mm}$ (width) $\times 1,320 \mathrm{~mm}$ (height) to eliminate the horizontal buoyancy that would otherwise be produced by the growth in boundary-layer displacement thickness. Although some wind tunnels do not employ corner fillets, the $150 \mathrm{~mm} \times 150 \mathrm{~mm}$ fillets at the end of the contraction were continued into the working section to reduce secondary flows and provide a convenient location for light fittings. Growing these fillets along the circuit (Table 1) also aided the design of the shape change module prior to the fan by producing an octagonal internal geometry. The length of the working section was set at approximately twice its hydraulic diameter to allow model wakes to mix out prior to the first diffuser $(1,10)$. The working section carcass was isolated from the rest of the tunnel structure by means of flexible couplings at inlet and exit, to minimise the transmission of structure borne vibration. The balance point of resolution was placed $1,695 \mathrm{~mm}$ downstream of the working section inlet. A safety screen of $D_{W}=2.5 \mathrm{~mm}$, and $\psi=$ $23 \mathrm{~mm}$, was located at the end of the working section. Due to the high porosity of the mesh, $K_{L}$ was calculated by treating each wire as an infinitely long cylinder and neglecting interaction effects between the wires.

\subsection{Diffusers}

Diffusers are used upstream of the corner modules to reduce the bulk flow velocity and hence losses associated with the turning vanes. A diffuser is placed behind the fan to recover as much of the pressure head as possible before the flow is dumped to atmosphere. Although the first and second diffusers are low aspect ratio plane-walled designs, they may be treated as conical diffusers since their total included angle $(2 \theta)$ is less than $8^{\circ}(15)$. This enables empirical performance charts $(15,16)$ developed for conical diffusers to be used in the design process.

The first diffuser has an area ratio $\left(A_{r}\right)$ of $1 \cdot 584$, a roof/floor total included angle $(2 \theta)$ of $5 \cdot 9^{\circ}$ and a sidewall $2 \theta$ of $5 \cdot 2^{\circ}$. Greater divergence was used in the vertical plane to reduce the aspect ratio of the internal circuit and thereby prevent the local wall angles in the constant-area shape-change module from being excessive. Since the performance of a diffuser is largely dependent upon the quality of its near wall flow, it was thought prudent to use a lower roof/floor $2 \theta$ than the theoretical maximum ${ }^{(16)}$ of $7^{\circ}$, as automotive models placed on the floor of the working section may produce non-uniform velocity profiles at inlet to the diffuser. The static pressure recovery coefficient,
$C p_{r}$, derived from published data ${ }^{(16)}$ is $0 \cdot 483$ and is defined as:

$C p_{r}=\frac{p_{2}-p_{1}}{1 / 2 \rho U_{1}^{2}}$

Where $p$ is the static pressure. The total pressure loss coefficient, $K_{L}$, for diffusers followed by a downstream duct is defined as ${ }^{(16)}$ :

$K_{L}=\alpha_{1}-\left(\frac{\alpha_{2}}{A_{r}^{2}}\right)-C p_{r}$

Where $A_{r}$ is the area ratio and $\alpha$, is the kinetic energy coefficient, which is a measure of the degree of flow non-uniformity. The latter is defined as:

$\alpha=\frac{1}{A U^{3}} \int_{A} u^{3} \mathrm{~d} A$

Given the wide variety of models that are likely to be tested, a very conservative value of $\alpha_{1}=1.2$ for the first diffuser, particularly as there was no risk (in terms of tunnel speed) associated in doing so.

The area ratio of the second diffuser is 1.384 , and it has a roof/floor $2 \theta$ of $7 \cdot 0^{\circ}$ (the theoretical maximum(16) since space was a severe constraint) and a sidewall $2 \theta$ of $5 \cdot 1$ degrees. Its $C p_{r}$, was obtained from published data $(16)$ as $0 \cdot 400$. Although the flow may still be non-uniform, an $\alpha_{1}=1.1$ was used since the wake produced by a model on the floor of the working section would have had time and space to mix out.

A conical rather than plane-walled diffuser is located downstream of the fan since it is better able to take advantage of the $5^{\circ}$ of swirl generated by the latter, and also because it eliminated the need for a second shape change module. The diffuser has an area ratio of 1.744 and a $2 \theta$ of $6 \cdot 0^{\circ}$. Its $C p_{r}$, was obtained from published data(16) as 0.538 and an $\alpha_{1}=1.1$ was adopted. Since the kinetic energy contained within the jet at exit of the final diffuser is not used in a downstream duct, the total pressure loss coefficient, $K_{L}$, is defined as(15):

$K_{L}=\alpha_{1}-C p_{r}$

An exit mesh identical to the working section safety screen, was placed at the end of the final diffuser to prevent foreign object ingress.

\subsection{Turning vanes}

Thin circular-arc turning vanes were used in both corners to guide the flow efficiently around each 90-degree bend. Previous investigations(17-19) have shown that vane space-to-chord ratios $(s / c)$ in the region of $0 \cdot 2-0 \cdot 25$ produce corner $K_{L}$ values of around $0 \cdot 12-0 \cdot 20$, compared to $K_{L}>1$ for the no vane case. Results from a vane test program that investigated various space to chord ratios (s/c), showed that an $s / c$ of $0 \cdot 190$ provided an acceptable near wall velocity distribution $25 \mathrm{~mm}$ and $875 \mathrm{~mm}$ downstream of the cascade and $K_{L}$ of $0 \cdot 128^{(20)}$. A conservative $K_{L}$ of $0 \cdot 15$ was used in the pressure loss calculations to account for the thicker boundary layer and model induced non-uniformity that may exist in the corners of the real wind tunnel. The wind tunnel has 25 vanes in the first corner and 28 in the second. All the vanes are of identical chord $(468.5 \mathrm{~mm})$ and profile and are made from $3 \mathrm{~mm}$ thick rolled aluminium. The underside of each vane is a concave circular arc of $245 \mathrm{~mm}$ radius, cut-off at the leading edge to produce a 4-degree angle-of-attack. The vanes have a trailing edge extension of $165 \mathrm{~mm}$ to encourage the flow to leave 
the vane axially, and roundness was applied to the leading edges to make them less sensitive to angularity in the incoming airstream. Although modern aerofoil sections have been proposed to further improve efficiency ${ }^{(17)}$, they were not considered for this wind tunnel since they have yet to be proved to be sufficiently insensitive to inlet flow angularity and were an order of magnitude more expensive to manufacture than circular arc vanes.

\subsection{Fan}

The 2.65m-diameter, variable-speed, fixed-pitch, fan was designed by Voith Howden. It has a hub to tip ratio of 0.42 and nine free vortex designed rotor blades. The motor is housed in the nacelle and integrally cooled through eight hollow straightener vanes. The fan was designed to allow for the later inclusion of louvres and exit guide vanes should they prove necessary, whilst still meeting the $40 \mathrm{~ms}^{-1}$ working section target velocity.

Whilst the previous sections have been concerned with the design process and performance prediction, the ones that follow will describe the measured performance of the wind tunnel in general and the working section in particular.

\subsection{COMPARISON OF PREDICTED AND MEASURED PERFORMANCE}

$K_{L}$ and $C p_{r}$ values were determined experimentally by using two rings of four wall static tappings located upstream and downstream of the module in question. The ends of the tappings were squared off and de-burred to ensure good quality readings. The wind tunnel was run with a clean working section at $40 \mathrm{~ms}^{-1}$ and a differential pressure transducer used to measure the averaged static pressure from each ring. Data was sampled at $1 \mathrm{kHz}$ for ten seconds and averaged over ten repeats to ensure confidence in the mean. A settling time of two minutes was allowed before sampling data since the tubing was necessarily long. Theoretical values of $K_{L}$ and $C p_{r}$ calculated using the methods described in Section 2.0 are compared to experimental values in Table 2. Local dynamic pressure, $q L$, is also quoted to enable the relative significance of the data to be assessed. Detailed discussion of the reasons for the differences between predicted and measured performance data is beyond the scope of this paper. It is intended that the performance of individual modules will be discussed in detail in subsequent papers. However, it is worth noting at this point that there clearly are differences between the way modules perform when part of a wind-tunnel system compared to data gathered from test rigs.

In addition to the modules listed in Table 2, model drag and working section skin friction can (in certain applications) make significant contributions to overall tunnel loss. However, in this wind tunnel, drag power for an automotive model with a drag coefficient of $0 \cdot 3$ at $5 \%$ blockage at $40 \mathrm{~ms}^{-1}$ was only $1 \cdot 5 \mathrm{~kW}$, and was therefore an insignificant component of the overall fan power requirement. Although working section skin friction was calculated as contributing $2 \cdot 3 \%$ to the overall tunnel loss, this is not included in Table 2 since an experimental value was not determined.

Having calculated an overall fan stagnation pressure rise $\Delta H$, The line power $P$ drawn by the fan may be computed using the equation below:

$$
P=\frac{\Delta H A_{w S} U_{W S}(R F)}{\eta_{\text {total }}}
$$

$$
\Delta H=\sum K_{L} q_{L}
$$

$\eta_{\text {total }}=\eta_{\text {fan }} \times \eta_{\text {motor }} \times \eta_{\text {controller }}$

Where $A_{W S}$ is the working section area, $R F$ is a reserve factor of $1 \cdot 1$ used to allow for additional losses through leaks and joints, and $\eta$ is efficiency. It may be seen that fan power is proportional to the cube of the working section velocity.

During commissioning, at an ambient pressure of $102,500 \mathrm{~Pa}$ and temperature of $9^{\circ} \mathrm{C}$, the clean tunnel achieved a maximum working

Table 2

\begin{tabular}{|c|c|c|c|c|c|}
\hline Module & $K_{L}$ Theory & $K_{L} \operatorname{Exp}$ & $C p_{r}$ Theory & $C p_{r} \operatorname{Exp}$ & $q_{L}(\mathrm{~Pa})$ \\
\hline Filter mesh & $3 \cdot 293$ & 4.948 & - & $F$ & 18 \\
\hline Screen1 + Honeycomb & $1 \cdot 266$ & 1.456 & F & F & 18 \\
\hline Screen 2 & $1 \cdot 010$ & 1.098 & - & F & 18 \\
\hline Safety screen & $0 \cdot 199$ & 0.266 & $F$ & F & 980 \\
\hline First diffuser & f- & - & 0.483 & 0.538 & 980 \\
\hline First corner & $0 \cdot 150$ & 0.113 & - & F & 380 \\
\hline Second diffuser & - & - & 0.400 & 0.446 & 380 \\
\hline Second corner & $0 \cdot 150$ & $0 \cdot 160$ & F & F & 200 \\
\hline Final diffuser & - & - & 0.538 & 0.684 & 200 \\
\hline Exit mesh & 0.199 & $0 \cdot 189$ & - & - & 65 \\
\hline
\end{tabular}

Theoretical and experimental $K_{L}$ and $C p_{r}$ and $q_{L}$ at $40 \mathrm{~ms}^{-1}$ 
section velocity of $46 \mathrm{~ms}^{-1}$, compared to a theoretical prediction (without louvres and exit guide vanes) of $44 \mathrm{~ms}^{-1}$ at the same ambient conditions. To represent a clean working section in the theoretical prediction, $\alpha_{1}=\alpha_{2}=1.04$ was used throughout except at inlet to the final diffuser where $\alpha_{1}$ was maintained at $1 \cdot 1$. The tunnel has an energy ratio, $E R$, of 1.42 defined as:

$$
E R=\frac{1}{\sum K_{L}\left(\frac{q_{L}}{q_{W S}}\right)}
$$

Although this energy ratio is three or four times lower than what might be expected for a closed circuit design, the tunnel is not a commercial facility intended to be run 24 hours a day, and so the life-cycle cost was considered secondary to the initial capital cost, particularly in light of the tight budget.

\subsection{WORKING SECTION FLOW QUALITY}

\subsection{Boundary layer}

It has been suggested(20) that the boundary-layer thickness, $\delta$, in a wind tunnel working section may be evaluated analytically by treating it's growth as that of a turbulent boundary layer growing over a flat plate of length, $x$, originating at the point of inflection of the contraction (location, $X$, in Fig. 3). One common equation for a turbulent boundary layer with a zero pressure gradient, $\delta$, is defined as ${ }^{(21)}$ :

$\frac{\delta}{x}=\frac{0 \cdot 38}{\operatorname{Re}_{x}^{0.2}}$

From Fig. 3 it may be seen that the distance between, $X$, and the measurement location, $A$, is $4,477 \mathrm{~mm}$, giving a predicted boundarylayer thickness of $65 \mathrm{~mm}$ at $40 \mathrm{~ms}^{-1}$. Figure 4 and Table 3 compare an experimentally determined boundary-layer profile, with that for a $65 \mathrm{~mm}$ thick Blasius boundary layer following a $1 / 7^{\text {th }}$ power law. The experimental profile was measured using a pitot static probe traversed in $5 \mathrm{~mm}$ increments. At each traverse position, data was sampled at $1 \mathrm{kHz}$ and averaged over 40,000 samples. The close agreement between the experimental and theoretical boundary-layer thickness $(\delta)$, displacement thickness $(\delta *)$ and momentum thickness $(\theta)$ shown in Table 3 suggests that the boundary-layer develops along the surface of the contraction without separation.

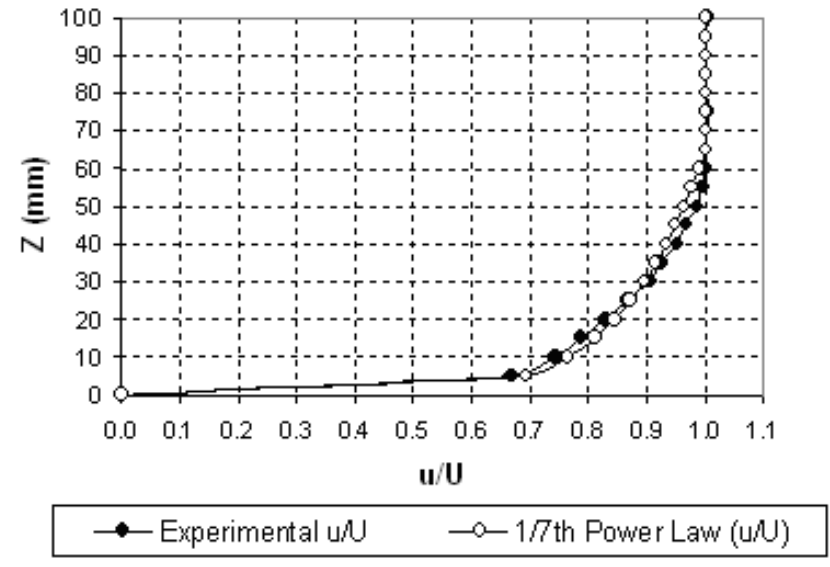

Figure 4. Centreline boundary-layer thickness at Station, A, on Fig. 3 at $40 \mathrm{~ms}^{-1}$.
Table 3

Comparison between theoretical and experimental boundary layer data

\begin{tabular}{|l|l|l|}
\hline & Theoretical & Experimental \\
\hline $\boldsymbol{\delta}(\mathbf{m m})$ & $65 \cdot 0$ & $60 \cdot 0$ \\
\hline $\boldsymbol{\delta} *(\mathbf{m m})$ & $7 \cdot 5$ & $9 \cdot 4$ \\
\hline $\boldsymbol{\theta}(\mathbf{m m})$ & 5.8 & 5.5 \\
\hline
\end{tabular}

\subsection{Velocity uniformity}

Figure 5 presents the velocity uniformity in the core of the working section and was experimentally determined using a pitot static probe traversed in an $80 \mathrm{~mm}$ grid, $100 \mathrm{~mm}$ in from the roof and floor of the working section. At each traverse position, dynamic pressure from the traversed probe (at station, A in Fig. 3) and the tunnel pitot static were recorded simultaneously. Data was sampled at $1 \mathrm{kHz}$ and averaged over 40,000 samples.

Figure 5 depicts the percentage variation from the mean axial velocity, $U$, at station $\mathrm{A}$ in Fig. 3. It may be seen that the velocity variation in the area normally occupied by floor mounted automotive models ( $Y \pm 250 \mathrm{~mm}, Z<350 \mathrm{~mm}$ ) deviates by around $0 \cdot 1 \%$ from the average. A more detailed assessment of the flow quality in this area will be made after installation of the boundary layer control system and moving ground. In the area normally occupied by aeronautical half models ( $Y \pm 100 \mathrm{~mm}, Z<1,000 \mathrm{~mm}$ ), velocity uniformity deviates by around $0 \cdot 3 \%$ from the average. In three localised areas outside of the model test area, velocity uniformity reaches $-0 \cdot 4 \%$. The concave velocity distribution produced at exit of a finite length contraction is shown by the velocity deficit in the core of the jet compared to the velocity overshoot around the perimeter.

\subsection{Turbulence intensity}

A single-wire hot-wire was used to measure the axial turbulence intensity at station, A, in Fig. 3 at $40 \mathrm{~ms}^{-2}$. The wire was calibrated using Dantec's Stream Line Constant Temperature Anemometry system and StreamWare application software. Data was sampled at $2 \mathrm{kHz}$, with a low pass filter set at $200 \mathrm{~Hz}$. 32,7680 samples were ensemble averaged over 40 blocks, giving a frequency resolution of $0 \cdot 244 \mathrm{~Hz}$.

Figure 6 shows that turbulence intensity decreases from around $3 \%$ at $z=60 \mathrm{~mm}$ (the edge of the boundary layer) to around $0 \cdot 15 \%$ in the core of the working section, which is close to the noise level of the instrument.

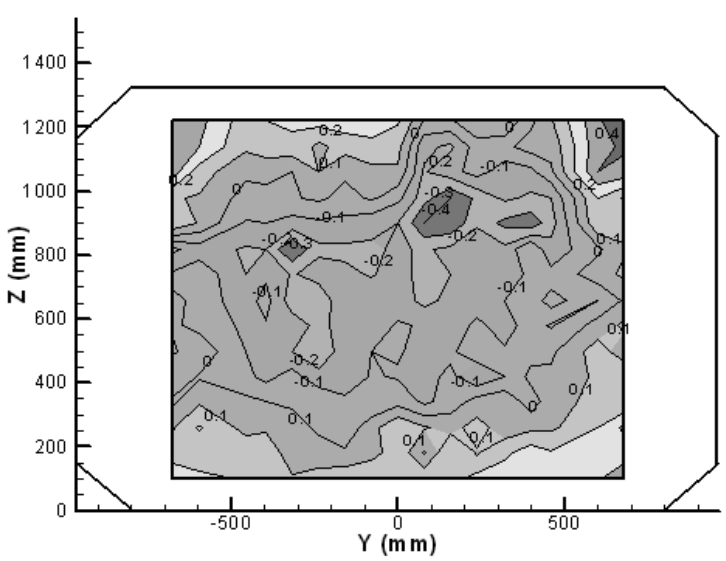

Figure 5. Percentage variation from mean velocity at Station A, Fig. 3 at $40 \mathrm{~ms}^{-1}$. 


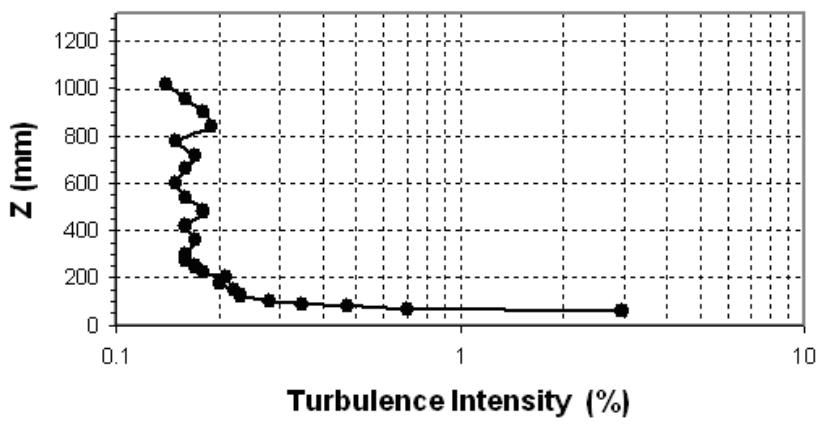

Figure 6. Centreline turbulence intensity at Station A on Fig. 3 at $40 \mathrm{~ms}^{-1}$.

\subsection{CONCLUSIONS}

Significant space and cost savings may be made by employing an indraft 'horseshoe' configuration as described in this paper, rather than a traditional closed circuit layout. Such a wind tunnel has been shown to deliver working section velocity uniformity within $0 \cdot 3 \%$ deviation from the mean velocity and a turbulence intensity of around $0 \cdot 15 \%$ at $40 \mathrm{~ms}^{-1}$, using a fine filter mesh (followed by a mixing length), honeycomb, two turbulence reduction screens and a contraction ratio of $7 \cdot 3$. The tunnel has the advantage of being able to run without the need for a heat exchanger. Working section boundary-layer characteristics have been shown to be consistent with that of a turbulent boundary layer growing along a flat plate, which originates at the point of inflection of the contraction. A maximum velocity of $46 \mathrm{~ms}^{-1}$ was achieved from a $140 \mathrm{~kW}$ motor, compared to a prediction of $44 \mathrm{~ms}^{-1}$. Comparison between theoretical and measured performance metrics indicate that there clearly are differences between the way modules perform when part of a wind tunnel system compared to data gathered from test rigs. After 18 months of use, the disadvantage of not being able to run the tunnel in heavy rain has not proved to be a serious problem, since model and instrumentation set-up periods and tunnel maintenance programmes can often be scheduled into such downtimes.

\section{ACKNOWLEDGEMENTS}

The authors would like to express sincere appreciation to Professor Stan Stevens, Dr Jonathan Cole, Dr Paul Denman, Dr Dachun Jiang, Mr Tony Eyre, Mr Grenville Cunningham, Mr Keith Coulthard, Mr Rob Hunter, Mr Norman Randall and Mr Peter Stinchcombe, without the efforts of whom the wind tunnel would not - in a very literal sense - exist!

\section{REFERENCES}

1. Barlow, J. Rae, W.H. and Pope, A. Low Speed Wind Tunnel Testing, 3rd ed, Wiley-Interscience, 1999.

2. Wolf, T. Improvement and modernization of subsonic wind tunnels, $J$ Aircr, 1993, 30, (1), pp 57-63.

3. Menta, R.D. and Bradshaw, P. Design rules for small low speed wind tunnels (Technical Note), Aeronaut J, 1979, pp 443-449.

4. Bradshaw, P and Pankhurst, R.C. The design of low speed wind tunnels, Progress in Aeron Sci, 1964, 5.

5. ESDU 80037, Pressure recovery of axisymmetric intakes at subsonic speeds.

6. LOEHRKE, R.I. and NaGiB, H.M. Experiments on management of freestream turbulence, (NATO) AGARD Report Number 598.

7. Schieman, J. Comparison of experimental and theoretical turbulence reduction characteristics for screens, honeycomb, and honeycombscreen combinations, NASA Technical Paper 1958, 1981.
8. Groth, J. and Johansson, A. Turbulence reduction by screens, J. Fluid Mech, 1988, 197, pp139-155.

9. Su, Y. Flow analysis and design of three-dimensional wind tunnel contractions, AIAA J, 1991, 29, (11), pp 1912-1919.

10. Pankhurst, R.C. and Holder, D.W. Wind Tunnel Technique, Pitman Press, 1968.

11. Morel, T. Comprehensive design of axisymmetric wind tunnel contractions, J Fluids Eng, 1975, pp 225-233.

12. TinkleR, J. and Fritz, E. Design of a 5:1 wind tunnel contraction, Canadian Aero and Space J, 1986, 32, (2), pp 108-112.

13. Downie, J.H., Jordinson, R. and Barnes, F.H. On the design of threedimensional wind tunnel contractions, Aeronaut J, 1984, pp 287-295.

14. ChMielewski, G.E. Boundary-Layer considerations in the design of aerodynamic contractions, J Aircr, 1974, 11, (8), pp 435-438.

15. ESDU 76027, Introduction to Design and Performance Data for Diffusers.

16. ESDU 73024, Performance of conical diffusers in incompressible flow.

17. Sahlin, A. and Johansson, A, Design of guide vanes for minimising the pressure loss in sharp bends, Phys Fluids, 1991, A, 3, (8), pp 19341940.

18. SALTER, C, Experiments on thin turning vanes; reports and memoranda No 2469, (Aerodynamics Division NPL), 25 October 1946.

19. WinTER, K.G. Comparative tests of thick and thin turning vanes in the Royal Aircraft Establishment 4 x 3ft wind tunnel, reports and memoranda No 2589, August 1947.

20. Johl, G.S., Passmore, M.A. and Render, P.M. Design and performance of wind tunnel turning vanes, (in preparation).

21. Larose, G.L. Tanguay, B. Van Every, D. and Bender T. The new boundary layer control system for NRC's 9m x 9m wind tunnel, AIAA2001-0455.

22. StReEt, R.L, Elementary Fluid Mechanics, John Wiley and Sons, 1996. 